\title{
On the Mohand Transform and Ordinary Differential Equations with Variable Coefficients
}

\author{
Mohamed E. Attaweel* and Haneen Almassry \\ Department of Mathematics, Arts \& Science Faculty, Gassr Khyar-Al Mergheb University, \\ Alkhomss, Libya
}

Received: 08 September 2019/ Accepted: 24 March 2020

Doi: https://doi.org/10.54172/mjsc.v35i1.229

\begin{abstract}
The Mohand transform is a new integral transform introduced by Mohand M. Abdelrahim Mahgoub to facilitate the solution of differential and integral equations. In this article, a new integral transform, namely Mohand transform was applied to solve ordinary differential equations with variable coefficients by using the modified version of Laplace and Sumudu transforms.
\end{abstract}

Keywords: Mohand Transform; Differential Equations.

\section{INTRODUCTION}

Integral transforms play an important role in many fields of science. In the literature, an integral transform is widely used in physics, astronomy, optics, and engineering mathematics.

The term "Differential Equation" was proposed in 1676 by Leibniz. The first studies of these equations were carried out in the late $17^{\text {th }}$ century. Differential equations are powerful tools in the study of many problems in science and technology (Khan et al., 2018).

Recently, Mohand M. Mahgoub introduces a new integral transform named the "Mohand Transform", and it has further applied to the solution of ordinary and partial differential equations. The purpose of this paper is to solve differential equations with variable coefficients using Mohand Transform.

\section{DEFINITIONS AND STANDARD RE- SULTS}

Definition 2.1 (Mohand Transform) (Mohand \& Mahgoub, 2017):

A new transform called the Mohand Transform is defined for a function of exponential order. We consider functions in the set A de- fined by

$$
A=\left\{\begin{array}{l}
f(t): \exists M, K_{1}, K_{2}>0,|f(t)|<M e^{\frac{|t|}{k_{j}}}, \\
\text { if } t \in(-1)^{j} \times[0, \infty)
\end{array}\right\}
$$

For a given function in the set $\mathrm{A}$, The constant $\mathrm{M}$ must be a finite number $k_{1}, k_{2}$ may be finite or infinite.

The Mohand Transform denoted by operator $\boldsymbol{M}(\cdot)$ defined by the integral:

$$
\begin{aligned}
& M[f(t)]=R(v)=v^{2} \int_{0}^{\infty} f(t) e^{-v t} d t \quad, t \geq 0, \\
& K_{1} \leq v \leq K_{2} \\
& \text { 1.1 Some Properties of Mohand Trans- } \\
& \text { form(Aggarwal \& Chauhan, 2019) }
\end{aligned}
$$

\subsection{Some Properties of Mohand Trans-}

a) Linearity Property :

if $M\left[f_{1}(t)\right]=R_{1}(v), M\left[f_{2}(t)\right]=R_{2}(v)$ then $M\left[a f_{1}(t)+b f_{2}(t)\right]=a R_{1}(v)+b R_{2}(v)$ where $a, b$ are arbitrary constants.

b) Change of Scale Property:

if $M[f(t)]=R(v)$ then

*Corresponding Author: Mohamed E. Attaweel, meattaweel@elmergib.edu.ly, Mathematics Department, Arts \& Science Faculty/ Gassr KhyarAl Mergheb University, Alkhomss, Libya 


$$
M[f(a t)]=a R\left(\frac{v}{a}\right)
$$

c) Shifting Property:

if $M[f(t)]=R(v)$ then

$$
M\left[e^{a t} f(t)\right]=\left[\frac{v^{2}}{(v-a)^{2}}\right] R(v-a)
$$

d) Convolution Theorem:

if $M\left[f_{1}(t)\right]=R_{1}(v), M\left[f_{2}(t)\right]=R_{2}(v)$ then

$$
M\left[f_{1}(t) * f_{2}(t)\right]=\frac{1}{v^{2}} R_{1}(v) \cdot R_{2}(v)
$$

where $f_{1}(t) * f_{2}(t)$ is defined by

$$
\begin{aligned}
f_{1}(t) * f_{2}(t) & =\int_{0}^{t} f_{1}(t-x) f_{2}(x) d x \\
& =\int_{0}^{t} f_{1}(x) f_{2}(t-x) d x
\end{aligned}
$$

e) Mohand Transform of the Integral of a Function:

$f(t):$ if $M[f(t)]=R(v)$ then

$M\left[\int_{o}^{t} f(t) d t\right]=\frac{1}{v} R(v)$

f) Mohand Transform of the Derivatives:

if $M[f(t)]=R(v)$, then

i) $M\left[f^{\prime}(t)\right]=v R(v)-v^{2} f(0)$

ii) $M\left[f^{\prime \prime}(t)\right]=v^{2} R(v)-v^{3} f(0)-v^{2} f^{\prime}(0)$

iii) $M[t f(t)]=\left[\frac{2}{v}-\frac{d}{d v}\right] R(v)$

iv) $M\left[t f^{\prime}(t)\right]=2 R(v)-2 v f(0)-\frac{d}{d v}$

$$
\left[v R(v)-v^{2} f(0)\right]
$$

v) $M\left[t f^{\prime \prime}(t)\right]=2 v R(v)-2 v^{2} f(0)-2 \times$

$$
\times v f^{\prime}(0)-\frac{d}{d v}\left[v^{2} R(v)-v^{3} f(0)-v^{2} f^{\prime}(0)\right]
$$

Notice that (i), (ii) are proved in (Mohand \& Mahgoub, 2017) and (iii) are proved in (Aggarwal \& Chauhan, 2019)
And from (iii), one can deduce that

$$
\begin{aligned}
M\left[t f^{\prime}(t)\right] & =\left[\frac{2}{v}-\frac{d}{d v}\right] M\left[f^{\prime}(t)\right] \\
& =\left[\frac{2}{v}-\frac{d}{d v}\right]\left[v R(v)-v^{2} f(0)\right] \\
& =2 R(v)-2 v f(0)-\frac{d}{d v}\left[v R(v)-v^{2} f(0)\right]
\end{aligned}
$$

And in a similar way, one can deduce that

$$
\begin{aligned}
& M\left[t f^{\prime \prime}(t)\right]=\left[\frac{2}{v}-\frac{d}{d v}\right] M\left[f^{\prime \prime}(t)\right] \\
& \quad=\left[\frac{2}{v}-\frac{d}{d v}\right]\left[v^{2} R(v)-v^{3} f(0)-v^{2} f^{\prime}(0)\right] \\
& =2 v R(v)-2 v^{2} f(0)-2 v f^{\prime}(0)-\frac{d}{d v}\left[v^{2} R(v)\right. \\
& \left.-v^{3} f(0)-v^{2} f^{\prime}(0)\right]
\end{aligned}
$$

g) Theorem 2.1: if $M[f(t)]=R(v)$ then

$$
\lim _{v \rightarrow \infty}\left[\frac{1}{v^{2}} R(v)\right]=0
$$

Proof:

$$
\begin{aligned}
& M[f(t)]=R(v)= v^{2} \int_{0}^{\infty} f(t) e^{-v t} d t \\
& \Rightarrow \frac{1}{v^{2}} R(v)=\int_{0}^{\infty} e^{-v t} f(t) d t \\
& \Rightarrow \lim _{v \rightarrow \infty}\left[\frac{1}{v^{2}} R(v)\right]=\lim _{v \rightarrow \infty} \int_{0}^{\infty} e^{-v t} f(t) d t \\
&=\int_{0}^{\infty} \lim _{v \rightarrow \infty} e^{-v t} f(t) d t \\
&=0
\end{aligned}
$$

$\therefore \lim _{v \rightarrow \infty}\left[\frac{1}{v^{2}} R(v)\right]=0$

Mohand Transform of Some Functions (Aggarwal et al., 2018) 


\begin{tabular}{|c|c|c|}
\hline S.N & $f(t)$ & $M[f(t)]=R(v)$ \\
\hline 1. & 1 & $v$ \\
\hline 2. & $t$ & 1 \\
\hline 3. & $t^{2}$ & $\frac{2 !}{v}$ \\
\hline 4. & $t^{n}, \quad n \in$ & $\frac{n !}{v^{n-1}}$ \\
\hline 5. & $e^{a t}$ & $\frac{v^{2}}{v-a}$ \\
\hline 6. & $\sin a t$ & $\frac{a v^{2}}{v^{2}+a^{2}}$ \\
\hline 7. & $\cos a t$ & $\frac{v^{3}}{v^{2}+a^{2}}$ \\
\hline 8. & $\sinh a t$ & $\frac{a v^{2}}{v^{2}-a^{2}}$ \\
\hline 9. & $\cosh a t$ & $\frac{v^{3}}{v^{2}-a^{2}}$ \\
\hline 10 & $J_{0}(t)$ & $\frac{v^{2}}{\sqrt{1+v^{2}}}$ \\
\hline 11. & $J_{0}(a t)$ & $\frac{v^{2}}{\sqrt{a^{2}+v^{2}}}$ \\
\hline 12. & $J_{1}(t)$ & $v^{2}-\frac{v^{3}}{\sqrt{1+v^{2}}}$ \\
\hline
\end{tabular}

\section{APPLICATIONS}

Example 3.1 (Khan et al., 2018)

Solve the differential equation:

$$
y^{\prime \prime}+t y^{\prime}-y=0
$$

with the initial condition,

$$
y(0)=0, y^{\prime}(0)=1
$$

Solution: Taking Mohand transform to give the following equation

$v^{2} R(v)-v^{3} f(0)-v^{2} f^{\prime}(0)+2 R(v)-2 v f(0)$

$-\frac{d}{d v}\left[v R(v)-v^{2} f(0)\right]-R(v)=0$

$v^{2} R(v)-v^{2}+R(v)-v R^{\prime}(v)-R(v)=0$

$R^{\prime}(v)-v R(v)=-v$ which is a linear differential equation. Its solution is

$$
\begin{aligned}
& R(v)=1+c e^{\frac{v^{2}}{2}} \\
& \Rightarrow R(v)=1 \quad(\text { by Theorem } 2.1 \quad c=0)
\end{aligned}
$$

By using inverse Mohand Transform, we get

$$
y(t)=t
$$

Example 3.2 (Nagle et al., 2014)

Consider the ordinary differential equation:

$$
y^{\prime \prime}+2 t y^{\prime}-4 y=1
$$

with the initial condition,

$$
y(0)=0, y^{\prime}(0)=0
$$

Solution: Taking Mohand transform to given equation

$$
\begin{aligned}
& v^{2} R(v)-v^{3} f(0)-v^{2} f^{\prime}(0)+4 R(v)-4 v f(0) \\
& -2 \frac{d}{d v}\left[v R(v)-v^{2} f(0)\right]-4 R(v)=v \\
& v^{2} R(v)-2 v R^{\prime}(v)-2 R(v)=v \\
& R^{\prime}(v)-\left[\frac{v^{2}-2}{2 v}\right] R(v)=\frac{-1}{2}
\end{aligned}
$$

Which is a linear differential equation. Its solution is

$$
R(v)=\frac{1}{v}+\frac{1}{v} c e^{\frac{v^{2}}{4}}
$$

$\Rightarrow \quad R(v)=\frac{1}{v} \quad($ by Theorem $2.1 \quad c=0)$

$\Rightarrow R(v)=\frac{1}{2 !}\left[\frac{2 !}{v}\right]$

By using inverse Mohand Transform, we get

$$
y(t)=\frac{1}{2} t^{2}
$$

Example 3.3 (Raisinghania, 2009)

Solve the differential equation: 


$$
t y^{\prime \prime}-y^{\prime}=t^{2}
$$

with the initial condition,

$$
y(0)=0, y^{\prime}(0)=0
$$

Solution: Taking Mohand transform to given equation

$$
\begin{aligned}
& 2 v R(v)-2 v^{2} f(0)-2 v f^{\prime}(0)-\frac{d}{d v}\left[v^{2} R(v)-\right. \\
& \left.v^{3} f(0)-v^{2} f^{\prime}(0)\right]-v R(v)+v^{2} f(0)=\frac{2}{v} \\
& v R(v)-2 v R(v)-v^{2} R^{\prime}(v)=\frac{2}{v} \\
& R^{\prime}(v)+\frac{1}{v} R(v)=\frac{-2}{v^{3}}
\end{aligned}
$$

Which is a linear differential equation. Its solution is

$$
R(v)=\frac{2}{v^{2}}+\frac{c}{v}
$$

By using inverse Mohand Transform, we get

$$
y(t)=\frac{1}{3} t^{3}+\frac{c}{2} t^{2}
$$

Example 3.4 (Raisinghania, 2009)

Solve the differential equation:

$$
t y^{\prime \prime}+y^{\prime}+4 t y=0
$$

with the initial condition,

$$
y(0)=3, y^{\prime}(0)=0
$$

Solution: Taking Mohand transform to given equation

$$
\begin{aligned}
& 2 v R(v)-2 v^{2} f(0)-2 v f^{\prime}(0)-\frac{d}{d v}\left[v^{2} R(v)-\right. \\
& \left.v^{3} f(0)-v^{2} f^{\prime}(0)\right]+v R(v)-v^{2} f(0)+ \\
& 4\left[\frac{2}{v}-\frac{d}{d v}\right] R(v)=0 \\
& 2 v R(v)-6 v^{2}-2 v R(v)-v^{2} R^{\prime}(v)+9 v^{2}+ \\
& v R(v)-3 v^{2}+\frac{8}{v} R(v)-4 R^{\prime}(v)=0
\end{aligned}
$$

$$
\begin{aligned}
& \left(v^{2}+4\right) R^{\prime}(v)-\left(v+\frac{8}{v}\right) R(v)=0 \\
& \Rightarrow R^{\prime}(v)=\left[\frac{v^{2}+8}{v\left(v^{2}+4\right)}\right] R(v) \\
& \Rightarrow \frac{d[R(v)]}{R(v)}=\frac{v^{2}+8}{v\left(v^{2}+4\right)}=\frac{\left(v^{2}+4\right)+4}{v\left(v^{2}+4\right)} \\
& \Rightarrow \frac{d[R(v)]}{R(v)}=\frac{2}{v}-\frac{v}{\left(v^{2}+4\right)} \\
& \Rightarrow R(v)=c \frac{v^{2}}{\sqrt{v^{2}+4}}
\end{aligned}
$$

By using inverse Mohand Transform, we get

$$
y(t)=c J_{0}(2 t)
$$

By using initial condition $y(0)=3$

Since $J_{0}(0)=1$, we get $c=3$

$$
\therefore y(t)=3 J_{0}(2 t)
$$

\section{CONCLUSION}

In this paper, we apply a new integral transform "Mohand Transform" to solve some ordinary differential equations with variable coefficients, and all solutions are satisfied by putting them back in the corresponding equations. The result reveals that the proposed method is very efficient, simple, and can be applied to linear differential equations.

\section{REFERENCES}

Aggarwal, S., \& Chauhan, R. (2019). A comparative study of Mohand and Aboodh transforms. International Journal of Research in Advent Technology, 7(1), 520-529.

Aggarwal, S., Chauhan, R., \& Sharma, N. (2018). Mohand transform of Bessel's 
functions. International Journal of Research in Advent Technology, 6(11), 3034-3038.

Khan, I. I., Khan, A. S. , and Khan, S. N. , (2018). On Mahgoub Transform and Ordinary Differential Equation with Variable Coefficient, International Journal of Scientific Research and Reviews , 7(4),51-54.ISSN 2279-0543

Mohand, M., \& Mahgoub, A. (2017). The new integral transform "Mohand Transform". Advances in Theoretical and Applied Mathematics, 12(2), 113120.

Nagle, R. K., Saff, E. B., \& Snider, A. D. (2014). Fundamentals of differential equations. Pearson.

Raisinghania, M. (2009). Advanced differential equations. S. Chand Publishing. 


\title{
مجلة المختار للعلوم 35 (1): 1-6، 2020
}

\section{حول تحويل مهند والمعادلات التفاضلية العادية ذات المعاملات المتغيرة}

\author{
محمد التاويل* وحنين المصري \\ قسم الرياضيات ، كلية الآداب و العلوم، قصر خبار -جامعة المرقب، مدينة الخدس ، ليييا
}

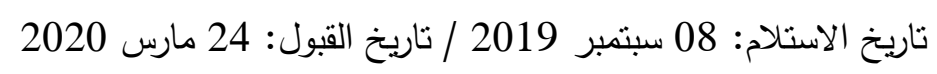
https://doi.org/10.54172/mjsc.v35i1.229:Doi

المستخلص: تحويل مهند هو تحويل تكاملي جديد قدمه مهند م. عبد الرحيم محجوب وذلك لتسيهيل حل المعادلات التفاضلية




الكلمات المفتاحية: تحويل مهند، معادلات تفاضلية. 